\title{
Multimodal interfaces and communication cues for remote collaboration
}

\author{
Seungwon Kim ${ }^{1} \cdot$ Mark Billinghurst ${ }^{1} \cdot$ Kangsoo Kim $^{2}$
}

Published online: 3 October 2020

(c) Springer Nature Switzerland AG 2020

\begin{abstract}
Remote collaboration has been studied for more than two decades and now there is the possibilities for new types of collaboration with the recent advances in immersive technologies such as Virtual, Augmented, Mixed Reality (VR/AR/MR). However, despite the increasing research interest in remote collaboration study with VR/AR/MR technologies, there is still a lack of academic venues specifically focusing on VR/AR/MR remote collaboration research. This special issue provides high-quality papers on the topic of remote collaboration research and increases visibility of this timely interesting and important research area. We particularly focus on three research aspects in remote collaboration: (1) use of multimodal communication cues, (2) awareness of the task space, and (3) human factors understanding. In this editorial, we first describe five essential factors for remote collaboration: task, local user, remote user, communication, and tool/interface, and then summarize a brief history of the research areas. We also cover the feature papers accepted in this issue, which introduce novel multimodal interfaces for remote collaboration and the effects on task performance and perceptual factors. Finally, we discuss some potential future research directions while concluding the editorial.
\end{abstract}

Keywords Remote Collaboration $\cdot$ Communication Cues $\cdot$ Multimodal Interfaces $\cdot$ Mixed Reality

\section{Introduction}

While our society and culture have been evolving, we have continuously developed skills to work/collaborate remotely with other people [46]. Here, collaboration is multi-person activities to achieve a common goal [12], and could be categorized into two sectors according to the collaborators' locations-e.g., whether they stay in the same place or not.

People usually have better collaboration when staying in the same place rather than when they are in remote places [22]. People intuitively and immediately shares

Kangsoo Kim

Kangsoo.Kim@ucf.edu

Seungwon Kim

Seungwon.Kim@unisa.edu.au

Mark Billinghurst

Mark.Billinghurst@unisa.edu.au

1 School of Information Technology and Mathematical Sciences, University of South Australia, GPO Box 2471, Adelaide, SA 5001, Australia

2 University of Central Florida, 3100 Technology Parkway, Orlando, FL 32826-3281, USA social/communication cues in co-located collaboration, but this is not always available in remote collaboration, especially with conventional remote communication technologies, e.g., telephone and videoconferencing tools.

Thanks to the recent advances in immersive technologies with multimodal interfaces, such as Virtual, Augmented, and Mixed Reality (VR/AR/MR) with support for gesture and gaze input, we encounter a new era of technologically enhanced remote collaboration with unique opportunities and challenges $[19,37]$. In remote collaboration studies, AR/VR/MR technologies have been used for visually showing spatial information in the real world task scene, and for providing an identical environment of the task scene to the remote collaborator who is not in the task space [2].

There have been a few research events related to this remote collaboration, for example, VR in VR: IEEE VR Workshop on Virtual Conferencing, 3DCVE: IEEE VR Workshop on 3D Collaborative Virtual Environments, MVAR: ISMAR Workshop on Multimodal Virtual \& Augmented Reality, and CoMiRE: ISMAR Workshop on Collaborative Mixed Reality Environments. However, there was a lack of academic journals, which could provide comprehensive yet novel findings 
and knowledge, particularly focusing on AR/VR/MR remote collaboration with multimodal input.

In this special issue, we compiled a collection of high quality contributions that reflect the latest state of the art of research in AR/VR/MR remote collaboration, and share research on multimodal interfaces and communication cues while providing insights for the future research. All submitted papers were peer-reviewed, and five accepted papers were selected based on their research quality and significance of the contribution to the existing literature. As an introduction to our special issue, this editorial describes the definition of the remote collaboration with five constructing factors, briefly summarizes the research aspects in remote collaborations, and introduces the five accepted feature papers. Finally, we will discuss some potential future research directions while concluding the editorial.

\section{Remote collaboration}

As mentioned earlier, collaboration can happen in different distance settings, either among co-located users in the same place (face to face collaboration), or between distributed users in remote places (remote collaboration). Remote collaboration has been studied in term of the collaborative performance and the user's perception for more than two decades in different research fields, such as telecommunication and social psychology. While there are dramatic advances in mobile and immersive technologies, e.g., VR/AR/MR, pervasive and personal mobile/wearable computing, the potential of such technologies is growing in collaboration tasks where multiple remote users are equipped with personal and immersive devices. Not surprising, research on remote collaboration is receiving more and more attention from not only the expert researchers and practitioners, but also public consumers, with the rapid development and enhancement of such technologies.

One of the common remote collaboration scenarios is a remote-expert and local-novice collaboration in different application contexts. The main purpose of this collaboration is to increase the availability of a remote expert, so that the local novice users could get help from the remote expert anywhere and anytime with appropriate technological supports. For example, a local novice might experience a broken machine that needs an expert's help repairing, a local doctor with little expertise might need to conduct an operation on a patient with the essential help from a distant expert surgeon, or a crime scene investigator could need help from a forensics expert in a remote place.

Here, we describe five essential factors for remote collaboration before addressing relevant research aspects in the following sections: (1) tasks, (2) local users, (3) remote users, (4) communication, and (5) tools for collaboration.
(1) Task: Since collaboration is a multi-person activity for a shared goal, there should be a task that collaborators are trying to complete together. A task often involves task objects that collaborators manipulate. Several researchers reported that the result of the remote collaboration can be task dependent because the required object manipulation could be different depending on the task type [28]. For example, a task for selecting an object most likely requires the object's position information shown with a pointing operation, but tasks like fixing a car or a bike require both position and orientation information for more complex object manipulation.

(2) Local user A local user is a person who is in the place where the task and the task objects are normally located [37]. The local user requests a help from a remote user who might have expert knowledge to complete the task. In a conventional setting like the remote-expert and local-novice scenario, the local user would manipulate task objects following the remote user's guidance.

(3) Remote user As opposed to the local user, a remote user is a person who provides information and guidance to the local user for completing the shared task [37]. The remote user normally does not stay at the place where the task objects are, but usually communicates with the local user to show or tell them what to do for the task.

(4) Communication Communication is the activity that the users perform to share their thoughts and intent while completing the task together. It involves using various communication cues, such as verbal and nonverbal behaviors, to establish common ground, improve the quality of communication, and eventually complete the task successfully. For example, the remote user may explain the solution to the local user using communication cues [27]. Verbal cues are usually considered as more fundamental and basic communication cues, but they have various limitations because of the lack of visual representations, which are often intuitive and efficient. Therefore, many remote collaboration systems employ visual communication cues, such as a pointer, sketching, and hand gestures, in addition to verbal communication.

(5) Awareness tool/interface Since the local and remote users are in different spaces, there should be a awareness tool (or an interface) to show the current state of the task and the activity progress [24]. Sharing the users' views is common feature of the collaboration interface, often using conventional video conferencing systems. The local and remote user's collaborative activities can be shown in the shared view(s), and communication cues also visually displayed. It is important to understand that the affordances of the collaboration tools can determine the quality of communication and so the general user experience and collaboration performance. 
Considering these five factors for remote collaboration, researchers have mainly studied three research aspects: (1) understanding of the effects of individual communication cues and the combinations of multimodal cues, (2) sharing the view of the task space for user awareness, and (3) human factors that include perception and cognition research and the investigation of user profiles. We will cover these three research aspects in the following sections.

\section{Communication cues and multimodalities}

Communication cues are a critical component for effective remote collaboration. Many researchers reported that it is more challenging to achieve better user experience and performance in remote collaboration compared to co-located collaboration. The main reason behind this is because of the lack of communication cues $[15,25]$. Therefore, researchers have been trying to develop novel remote collaboration systems that could offer richer and more efficient communication cues for the users to easily understand the task situation, while understanding the effects of those cues [9,32].

With typical video conferencing systems, researchers and practitioners started to add additional visual cues onto the live video stream of the task space, to provide richer communication channels, such as a visual pointer, sketches, and the users' hand gestures [9]. Due to the technical challenge of capturing dynamic gestures of the users, the visual communication cues used in early remote collaboration systems were generally limited to a simple pointer and sketches. Later, Kirk et al. [31] implemented an system that could simply capture and share a live video of hand gestures. However, the cues were still primitive pointers, sketches, and hand gestures in $2 \mathrm{D}$, and the system had limited portability with a fixed camera on a tripod and a monitor display. It also did not support any sophisticated computer vision tracking, which caused difficulty to visualize the cues robustly even when the camera had moderate motions. While addressing this issue for pointer and sketch cues, Kato and Billinghurst [18] used vision-based tracking for stabilizing the sketches-the sketches were at the position where they were drawn regardless of the view changes. Kim et al. [30] also developed a system that significantly increased portability by using a handheld tablet, and Gauglitz et al. [10] and Kim et al. [28] further enhanced both portability and sketch stabilization. Regarding hand gesture cues, Alem et al. [1] increased the portability for a local worker wearing lightweight glasses, and Huang et al. [14] introduced a system that could capture and share the user's hand gestures and local task space in 3D.

Recently, there are three trends appearing in the study of the communication cues. First, researchers have started more focusing on the use of gaze cues as an effective communica- tion cue $[6,38]$, especially given the enhanced performance and popularity of low cost eye trackers. By using the gaze cues, the users in local and/or remote places can identify where the collaboration partner is attending and understand the task state and the partner's intentions. Gupta et al. [11] developed a system that visualizes the local user's gaze as a pointer on the shared view, while Higuchi et al. [13] showed the remote users' gaze pointers. Lee et al. [35] chose a bidirectional gaze sharing approach that adds both remote and local users' gaze pointers on the shared view.

Second, it is becoming more and more popular to show multiple visual cues simultaneously in remote collaboration. While individual visual cues have their own benefits and drawbacks, the combination of them could offer an opportunity to have richer social interactions while complementing each other. For example, Huang et al. [15] explored the use of the combined communication cues using sketch and hand gesture cues.

Third, in addition to the multiple visual cues, combining different sensory modalities for communication is gaining attention from multimodal interfaces and remote collaboration researchers. For example, DeVincenzi et al. [4] combined a spatial audio cue, the remote user's spatialized voice, together with a live video stream, and found that the local user could more easily identify where the remote user was located by the direction of the audio source.

\section{View sharing and situational awareness}

Another important aspect that influences the collaboration experience in remote settings is to share the user's view with the collaboration partner. For example, the local user's view can be shared with the remote expert so that he/she can understand the task progress by watching the shared view $[16,34]$. This sharing view feature is particularly important because the users could not only see the current state of the task, but they could also put themselves in another person's shoes. In collaboration, social understanding and grounding are crucial for developing better communication and rapport among the collaborators [32]. The view sharing methods are advancing both quantitatively and qualitatively, for example use of high definition shared views, large panoramic views, and immersive technology like first-person VR experience [44].

In the early stage of remote collaboration technologies, researchers used a video conferencing system that could only show a simple live video of the task space [9] and the live video was streamed from a fixed camera on the local user's head or environment [29]. With a handheld tablet, it was not convenient to share the local user's perspective with the remote users because the user's hands should be occupied by holding the tablet/camera while they also need to perform the given task [8]. Recent compact wearable camera systems, 
such as GoPro cameras [41], or see-through head-mounted displays (HMDs) with embedded cameras, like Microsoft HoloLens, could free the user's hands and make view sharing simple and easy.

While the shared view or the layout can be independently controlled by the remote/local user, some researchers stitched the images from a live video and then constructed a large image covering the overall task space $[10,17]$. With the large image, the remote expert could have an independent view regardless of the current viewpoint of local user's live video and freely navigate around the task space. For example, Lee et al. [36] introduced a system sharing a 360-degree live video, so both local and remote users could individually control the viewpoint in the 360-degree simulated environment. Piumsomboon et al. [42] also developed a system that can share a $3 \mathrm{D}$ reconstructed environment of the local user's task space and support both local and remote users' navigation in the environment.

\section{Human factors}

In remote collaboration contexts, the users should be able to interact with the remote collaborators in different places both effectively and efficiently for best task performance. However, the overall user experience regarding their feeling of ease of communication, understanding of the other users' emotional states, the sense of social/co-presence and comfort is also important to evaluate when considering the effectiveness of the collaboration systems. Thus, while developing novel interfaces and effective tools for remote collaborations, human factors research to understand perception, cognition, and behavior with the technology should be conducted and supported.

Researchers have actively studied how visual cues and tools of sharing a task space influence the collaborators' sense of social/co-presence and cognitive load during the collaboration. For instance, Kim et al. [28] conducted a study that investigated the effects of augmented visual cues on the user-perceived co-presence with the remote user. Piumsomboon et al. [43] compared different sharing view modes, such as shoulder-mounted or hand-held camera views, and found that users generally preferred the shoulder-mounted view and reported a higher level of co-presence when there was a shared view. Regarding the effects of visual cues, Huang et al. [15] found that local and remote users have lower cognitive load during a remote collaboration when they had more visual communication cues available. More recently, Dey et al. [5] studied the effect of sharing the user's physiological signals with the remote users, such as heart beat as a signal for representing the user's emotional state, and found a positive influence for emotional experience in a gaming context.

\section{Featured work}

In this section, we introduce the five accepted papers in this issue and briefly summarize their results and findings, in the context of the three research aspects; communication cues and multimodalities, view sharing and situational awareness, and human factors.

The combination of visual communication cues in mixed reality remote collaboration In this paper (Kim et al. [26]), explores the impact of combining different visual cues on the user-perceived quality of communication and collaboration performance. The visual cues were a pointer, sketches, and hand gesture cues, which are traditionally independent in remote collaboration.

Two conducted user studies compared four combinations of the three cues: (1) hand only, (2) hand+pointer, (3) hand+sketch, and (4) hand + pointer + sketch, while varying the sharing view mode, either a dependent or an independent view. In the dependent view mode, in which the users shares the same view, participants mostly used the hand gestures due to ease of use and intuitiveness, while the sketches were also useful for clarifying any misunderstandings between the users. Interestingly, in the independent view mode, in which the users had their own views, the hand gesture cues were perceived difficult to identify where the hand referred to, because of the different perspectives of the users. However, the pointer cue turned out to be very useful as an alternative for the hand gestures.

The effects of spatial auditory and visual cues on mixed reality remote collaboration: Despite the importance of auditory cues in remote collaboration, the current state of research in MR remote collaboration largely focuses on investigating the effects of visual cues. To fill the gap, this paper (Yang et al. [47]), presents a multimodal system that can provide both spatial auditory and visual cues to the users, and investigates the effects of such multimodal cues on task performance and perception in an indoor visual search task through. The user study results show that the remote user's spatialized voice guidance and auditory beacons could improve the local user's performance to find objects in a highly cluttered environment by providing auditory clues for spatial directions. The paper further discusses the potential implications in the integration of spatial auditory and visual cues for better user experience and performance in remote collaboration contexts.

Sharing gaze rays for visual target identification tasks in collaborative augmented reality: Sharing social cues among collaborators is a powerful strategy to improve the quality of communication. In this paper (Erickson et al. [7]), authors explore the use of a shared gaze ray in a target identification task while investigating the influence of different gaze errors in the task performance, such as response time and error rate. 
The results show that different error levels in the shared gaze had strong effects on participants' performance, while the distance to the task object had less influence on the performance and user experience. Interestingly, the study also reveals that participants' self-assessed performance was lower than the actual performance in the target identification task.

Exploring interaction techniques for 360 panoramas inside a $3 D$ reconstructed scene for mixed reality remote collaboration: In this paper (Teo et al. [45]), authors investigate the effects of different ways to show 360-degree live video on the user's sense of social/co-presence and cognitive load in collaborative object moving tasks. The conducted user study compared two modes: (1) projecting the 360-degree live video in a sphere, called the "photo-bubble" mode, and (2) projecting it as a texture on a low-resolution 3D reconstructed mesh, called the "projective texture" mode. The paper presents the results suggesting that both modes could provide a high level of social/co-presence and reduce cognitive load, and discusses the advantages and limitations of each method.

Effects of personality traits on user trust in human-machine collaborations: Beyond collaborations between human users, there is increasing research in human-machine (or humanagent) collaborations, considering the convergence of advanced artificial intelligence (AI), such as intelligent virtual assistants, and immersive AR/VR technologies [39]. This paper Zhou et al. [48] explores the effect of a user's personality trait on the level of trust in a machine's decisions when collaborating with the machine. The user study conducted in the paper varied the task design in terms of the levels of uncertainty and cognitive load, and examined how participants with different personality traits perceived the trustworthiness of the collaborative machine's decisions while performing the task. The results reveals that the users' personality traits affect their perceived trust in machine decision differently with respect to the uncertainty and cognitive load conditions.

\section{Conclusion and future work}

To provide a comprehensive background and share recent significant findings in remote collaboration research, we prepared this Special Issue on Multimodal Interfaces and Communication Cues for Remote Collaboration, while focusing on but not necessarily limited to immersive technologies like AR/VR/MR. This editorial described the concept of remote collaboration and the research aspects of multimodal communication cues, sharing the users' views, and related human factors for the reader's understanding of the focused research, and the summaries of the accepted papers written by the domain-expert researchers and students were provided.

While technologies for remote collaboration are evolving even beyond the limited scope of "remote," the research aiming at better collaboration and communication tools is anticipated to be growing in the future. Despite the good amount of research on collaboration-support technologies, there are still a lot of gaps that need to be filled by researchers and practitioners. For example, regarding the multimodal communication cues, the use of multimodal cues could be extended to various sensory modalities, such as haptics and olfactory [23]. The use of communication cues could also go beyond the scope of human sensory channels, e.g., interpretable brain signals through electroencephalogram (EEG), which are already actively researched in brain-computer interface [33].

The design of the communication cues can also be adaptively changed according to user needs. For instance, when using hand gesture cues in a large task space where such small gestures could be difficult to understand due to the far distance from a target object, the hand gesture cue can be transferred on the object in a different form, such as a magnified gesture cue or a virtual pointer [3]. The pointer cue can have different size, color, and level of transparency according to distance to the task objects, and appropriate changes of the pointer in color and size can moderate the balance between attracting the user's attention and reducing the disturbance to see the target objects during the task.

Considering the recent popularity of social VR platforms, embodied virtual avatars or agents could also be an effective approach to support or replace human users in collaboration tasks [20,21], and research to understand the effects of different avatar shapes/appearances in AR/VR is already in progress [40].

For the immersive and realistic view sharing, there is still no practical implementation for generating and sharing finegrained 3D reconstruction of the dynamic task space in real time. In the future, various convergence research should be conducted to achieve this using advanced computer vision, data compression, and network techniques, while the perception and cognition studies continue to use different measures and tools as a broader scope of human-computer interaction and human factors research.

\section{References}

1. Alem L, Tecchia F, Huang W (2011) Remote tele-assistance system for maintenance operators in mines. In: 11th Underground coal operators' conference. University of Wollongong

2. Baecker RM (1994) Readings in groupware and computersupported cooperative work: assisting human-human collaboration, 1st edn. Morgan Kaufmann, San Francisco 
3. Choudhary Z, Kim K, Schubert R, Bruder G, Welch GF (2020) Virtual big heads: analysis of human perception and comfort of head scales in social virtual reality. In: IEEE conference on virtual reality and 3D user interfaces, pp 425-433

4. DeVincenzi A, Yao L, Ishii H, Raskar R (2011) Kinected conference: augmenting video imaging with calibrated depth and audio. In: Proceedings of the ACM 2011 conference on computer supported cooperative work, pp 621-624

5. Dey A, Piumsomboon T, Lee Y, Billinghurst M (2017) Effects of sharing physiological states of players in a collaborative virtual reality gameplay. In: Proceedings of the $2017 \mathrm{CHI}$ conference on human factors in computing systems, pp 4045-4056

6. Erickson A, Norouzi N, Kim K, LaViola JJ, Bruder G, Welch GF (2020) Effects of depth information on visual target identification task performance in shared gaze environments. IEEE Trans Visu Comput Graph 26(5):1934-1944

7. Erickson A, Norouzi N, Kim K, Schubert R, Jules J, LaViola JJ, Bruder G, Welch GF (2020) Sharing gaze rays for visual target identification tasks in collaborative augmented reality. J Multimodal User Interfaces. https://doi.org/10.1007/s12193-020-00330-2

8. Fakourfar O, Ta K, Tang R, Bateman S, Tang A (2016) Stabilized annotations for mobile remote assistance. In: Proceedings of the $2016 \mathrm{CHI}$ conference on human factors in computing systems, pp $1548-1560$

9. Fussell SR, Setlock LD, Yang J, Ou J, Mauer E, Kramer ADI (2004) Gestures over video streams to support remote collaboration on physical tasks. Hum-Comput Interact 19(3):273-309

10. Gauglitz S, Nuernberger B, Turk M, Höllerer T (2014) Worldstabilized annotations and virtual scene navigation for remote collaboration. In: Proceedings of the 27th annual ACM symposium on user interface software and technology, pp 449-459

11. Gupta K, Lee GA, Billinghurst M (2016) Do you see what i see? The effect of gaze tracking on task space remote collaboration. IEEE Trans Vis Comput Graph 22(11):2413-2422

12. Gutwin C, Greenberg S (1998) Design for individuals, design for groups: tradeoffs between power and workspace awareness. In: Proceedings of the ACM conference on computer supported cooperative work, pp 207-216

13. Higuch K, Yonetani R, Sato Y (2016) Can eye help you? Effects of visualizing eye fixations on remote collaboration scenarios for physical tasks. In: Proceedings of the $2016 \mathrm{CHI}$ conference on human factors in computing systems, pp 5180-5190

14. Huang W, Alem L, Tecchia F, Duh HBL (2018) Augmented 3D hands: a gesture-based mixed reality system for distributed collaboration. J Multimodal User Interfaces 12(2):77-89

15. Huang W, Kim S, Billinghurst M, Alem L (2019) Sharing hand gesture and sketch cues in remote collaboration. J Vis Commun Image Represent 58:428-438

16. Irlitti A, Piumsomboon T, Jackson D, Thomas BH (2019) Conveying spatial awareness cues in xR collaborations. IEEE Trans Visu Comput Graph 25(11):3178-3189

17. Kasahara S, Rekimoto J (2014) JackIn: integrating first-person view with out-of-body vision generation for human-human augmentation. In: Proceedings of the 5th augmented human international conference, pp 46:1-46:8

18. Kato H, Billinghurst M (1999) Marker tracking and HMD calibration for a video-based augmented reality conferencing system. In: Proceedings 2nd IEEE and ACM international workshop on augmented reality (IWAR'99), pp 85-94

19. Kim K, Billinghurst M, Bruder G, Duh HBL, Welch GF (2018) Revisiting trends in augmented reality research: a review of the 2nd decade of ISMAR (2008-2017). IEEE Trans Vis Comput Graph 24(11):2947-2962

20. Kim K, Boelling L, Haesler S, Bailenson JN, Bruder G, Welch GF (2018) Does a digital assistant need a body? The influence of visual embodiment and social behavior on the perception of intelligent virtual agents in AR. In: IEEE international symposium on mixed and augmented reality, pp 105-114

21. Kim K, de Melo C, Norouzi N, Bruder G, Welch G (2020) Reducing task load with an embodied intelligent virtual assistant for improved performance in collaborative decision making. In: Proceedings of the IEEE conference on virtual reality and 3D user interfaces, pp 529-538

22. Kim K, Norouzi N, Losekamp T, Bruder G, Anderson M, Welch G (2019) Effects of patient care assistant embodiment and computer mediation on user experience. In: Proceedings of IEEE international conference on artificial intelligence and virtual reality, pp $17-24$

23. Kim K, Schubert R, Hochreiter J, Bruder G, Welch G (2019) Blowing in the wind: increasing social presence with a virtual human via environmental airflow interaction in mixed reality. Comput Graph $83: 23-32$

24. Kim S, Billinghurst M, Lee C, Lee G (2018) Using freeze frame and visual notifications in an annotation drawing interface for remote collaboration. KSII Trans Internet Inf Syst 12(12):6034-6056

25. Kim S, Billinghurst M, Lee G (2018) The effect of collaboration styles and view independence on video-mediated remote collaboration. Comput Support Cooper Work 27(3):569-607

26. Kim S, Lee G, Billinghurst M, Huang W (2020) The combination of visual communication cues in mixed reality remote collaboration. $\mathrm{J}$ Multimodal User Interfaces. https://doi.org/10.1007/s12193-02000335-x

27. Kim S, Lee G, Huang W, Kim H, Woo W, Billinghurst M (2019) Evaluating the combination of visual communication cues for HMD-based mixed reality remote collaboration. In: Proceedings of the $2019 \mathrm{CHI}$ conference on human factors in computing systems, pp 1-13

28. Kim S, Lee G, Sakata N, Billinghurst M (2014) Improving copresence with augmented visual communication cues for sharing experience through video conference. In: Proceedings of the IEEE international symposium on mixed and augmented reality, pp 83 92

29. Kim S, Lee GA, Ha S, Sakata N, Billinghurst M (2015) Automatically freezing live video for annotation during remote collaboration. In: Proceedings of the 33rd annual ACM conference extended abstracts on human factors in computing systems, pp 1669-1674

30. Kim S, Lee GA, Sakata N (2013) Comparing pointing and drawing for remote collaboration. In: Proceedings of the IEEE international symposium on mixed and augmented reality, pp 1-6

31. Kirk D, Stanton Fraser D (2006) Comparing remote gesture technologies for supporting collaborative physical tasks. In: Proceedings of the SIGCHI conference on human factors in computing systems, pp 1191-1200

32. Kraut RE, Gergle D, Fussell SR (2002) The use of visual information in shared visual spaces: informing the development of virtual co-presence. In: Proceedings of the 2002 ACM conference on computer supported cooperative work, pp 31-40

33. Lecuyer A, Lotte F, Reilly R, Leeb R, Hirose M, Slater M (2008) Brain-computer interfaces, virtual reality, and videogames. Computer 41(10):66-72

34. Lee G, Kang HY, Lee JM, Han JH (2020) A user study on viewsharing techniques for one-to-many mixed reality collaborations. In: Proceedings of the IEEE conference on virtual reality and 3D user interfaces, pp 343-352

35. Lee G, Kim S, Lee Y, Dey A, Piumsomboon T, Norman M, Billinghurst $M$ (2017) Mutually shared gaze in augmented video conference. In: Adjunct proceedings of the 2017 IEEE international symposium on mixed and augmented reality-adjunct, pp 79-80

36. Lee G, Teo THL, Kim S, Billinghurst M (2018) A user study on mr remote collaboration using live 360 video. In: Proceedings of the IEEE international symposium for mixed and augmented reality, pp 153-164 
37. Lukosch S, Billinghurst M, Alem L, Kiyokawa K (2015) Collaboration in augmented reality. Comput Support Cooper Work 24(6):515-525

38. Norouzi N, Erickson A, Kim K, Schubert R, Laviola Jr, JJ, Bruder G, Welch GF (2019) Effects of shared gaze parameters on visual target identification task performance in augmented reality. In: Proceedings of ACM symposium on spatial user interaction, pp 12:1-12:11

39. Norouzi N, Kim K, Hochreiter J, Lee M, Daher S, Bruder G, Welch $G$ (2018) A systematic survey of 15 years of user studies published in the intelligent virtual agents conference. In: Proceedings of the ACM international conference on intelligent virtual agents, pp 1722

40. Norouzi N, Kim K, Lee M, Schubert R, Erickson A, Bailenson J, Bruder G, Welch G (2019) walking your virtual dog: analysis of awareness and proxemics with simulated support animals in augmented reality. In: Proceedings of IEEE international symposium on mixed and augmented reality, pp 253-264

41. Paro JA, Nazareli R, Gurjala A, Berger A, Lee GK (2015) Videobased self-review. Ann Plast Surg 74:S71-S74

42. Piumsomboon T, Lee GA, Hart JD, Ens B, Lindeman RW, Thomas BH, Billinghurst M (2018) Mini-Me: an adaptive avatar for mixed reality remote collaboration. In: Proceedings of the $2018 \mathrm{CHI}$ conference on human factors in computing systems, pp 1-13

43. Piumsomboon T, Lee GA, Irlitti A, Ens B, Thomas BH, Billinghurst M (2019) On the shoulder of the giant: a multi-scale mixed reality collaboration with 360 video sharing and tangible interaction. In: Proceedings of the $2019 \mathrm{CHI}$ conference on human factors in computing systems, pp 1-17
44. Teo T, Lawrence L, Lee GA, Billinghurst M, Adcock M (2019) Mixed reality remote collaboration combining 360 video and 3D reconstruction. In: Proceedings of the ACM CHI conference on human factors in computing systems, vol 201. ACM Press, New York, pp 1-14

45. Teo T, Norman M, Lee GA, Billinghurst M, Adcock M (2020) Exploring interaction techniques for 360 panoramas inside a 3D reconstructed scene for mixed reality remote collaboration. J Multimodal User Interfaces. https://doi.org/10.1007/s12193-02000343-x

46. Tomasello M (2016) Precís of a natural history of human thinking. J Soc Ontol 2(1):59-64

47. Yang J, Sasikumar P, Bai H, Barde A, Sörös G, Billinghurst M (2020) The effects of spatial auditory and visual cues on mixed reality remote collaboration. J Multimodal User Interfaces. https:// doi.org/10.1007/s12193-020-00331-1

48. Zhou J, Luo S, Chen F (2020) Effects of personality traits on user trust in human-machine collaborations. J Multimodal User Interfaces. https://doi.org/10.1007/s12193-020-00329-9

Publisher's Note Springer Nature remains neutral with regard to jurisdictional claims in published maps and institutional affiliations. 